\title{
gु Scaling behavior of coarsening Faraday heaps
}

\author{
Henk Jan van Gerner, ${ }^{1,2}$ Ko van der Weele, ${ }^{3}$ Devaraj van der Meer, ${ }^{1}$ and Martin A. van der Hoef ${ }^{1}$ \\ ${ }^{1}$ Faculty of Science and Technology, University of Twente, NL-7500 AE Enschede, The Netherlands \\ ${ }^{2}$ National Aerospace Laboratory, P.O. Box 153, NL-8300 AD Emmeloord, The Netherlands \\ ${ }^{3}$ Department of Mathematics, University of Patras, 26500 Patras, Greece
}

(Received 28 May 2015; published 16 October 2015)

\begin{abstract}
When a layer of sand is vertically shaken, the surface spontaneously breaks up in a landscape of small conical "Faraday heaps," which merge into larger ones on an ever increasing time scale. We propose a model for the heap dynamics and show analytically that the mean lifetime of the transient state with $N$ heaps scales as $N^{-2}$. When there is an abundance of sand, such that the vibrating plate always remains completely covered, this means that the average diameter of the heaps grows as $t^{1 / 2}$. Otherwise, when the sand is less plentiful and parts of the plate get depleted during the coarsening process, the average diameter of the heaps grows more slowly, namely as $t^{1 / 3}$. This result compares well with experimental observations.
\end{abstract}

DOI: 10.1103/PhysRevE.92.042203

PACS number(s): 45.70.-n, 05.65.+b, 47.11.-j

\section{INTRODUCTION}

When a flat layer of fine sand is brought into vertical vibration, an intriguing phenomenon occurs: The flat surface swiftly breaks up into many small heaps, which then on a slower time scale merge together (forming fewer but larger heaps) until finally they are all united in one large heap, which thereafter remains stable. The first detailed study of a stable single heap on a vibrating plate was performed by Faraday in 1831 [1], and the phenomenon is therefore often called "Faraday heaping." Although Faraday's paper mainly focuses on the Chladni patterns on a resonating plate (coarse particles collect at the nodes, finer particles at the antinodes), towards the end he describes the peculiar motion of the particles within a single heap: "When a single heap is examined,..., it will be seen that the particles of the heap rise up at the center, overflow, fall down upon all sides, and disappear at the bottom, apparently proceeding inwards." Faraday concluded that the flow of air-induced by the partial vacuum that is created under the heap during the second part of a vibrational cycle-was responsible for this motion. This mechanism was later verified and analyzed in detail by the experiments of Thomas and Squires [2] and the numerical simulations of Van Gerner et al. [3]. In addition, Faraday also mentioned the key steps in the evolution of a flat surface into a single heap: "As to their first formation, the slightest irregularities in (..) the surface would determine a commencement, which would then instantly favour the increase" and "when two or more heaps are near together or touch, they will frequently coalesce and form but one heap, which quickly acquires a rounded outline."

While the case of a stable single Faraday heap is wellstudied [4-9], the collective dynamics of a number of them (i.e., the coarsening process) has received relatively little attention. To our knowledge there have been two studies on the subject in a restricted, quasi-two-dimensional setup $[10,11]$ and only one publication on the full problem [12], which is remarkable, since the merging of Faraday heaps is one of the clearest and most beautiful examples of coarsening in granular matter. It is not the only system in which the interplay of sand and the surrounding medium gives rise to patterns that coarsen over time: Other classic examples are underwater sand ripples at the beach [13-18] and wind-driven barchan dunes in the deserts on Earth and Mars [19-25], yet in those cases the coarsening process is never seen to run its full course. The growth of beach ripples and barchan dunes is limited by various factors and the coarsening process leads to the selection of a preferred length scale rather than to the formation of one big ripple or giant dune. By contrast, Faraday heaping generally does not end before all small humps have merged into one single heap.

In a broader context, the phenomenon fits into the general field of dynamical systems out of equilibrium, which are renowned for their susceptibility to spontaneous pattern formation [26-28]. Coarsening is one of the principal routes by which patterns are known to ripen to their final form, and Faraday heaping provides a particularly pure example of this.

A characteristic feature of coarsening is that it takes place on an ever increasing time scale. In the case of Faraday heaping this means that the time it takes to proceed from $N$ to $N-1$ heaps is longer than the previous step from $N+1$ to $N$ heaps, and one of the central questions is how this process scales with time.

In a previous paper we developed an analytical model, based on experiments and detailed numerical observations, to study the coarsening process in a quasi-two-dimensional setup where the sand was confined between two vertical glass plates [11]. In the simulations the motion of the sand particles was determined via molecular dynamics techniques, while the flow of the air was calculated by computational fluid dynamics. These simulations guided us to construct a coupled set of equations of motion for the $N$ peak positions, which were solved numerically and proved to accurately describe the evolution of the heap landscape in the quasi-twodimensional experiment [11]. In the present paper we extend the model to true three-dimensional (3D), as in Faraday's classic experiment, and the resulting scaling behavior is compared with the experimental findings of Shinbrot [12]. As a preview, in Fig. 1 we show a typical run of Shinbrot's experiment (top row) together with representative snapshots from our model (bottom row).

\section{MODEL FOR THE COARSENING PROCESS}

\section{A. On the formation of the landscape of heaps}

For a proper understanding of the model, it is useful to first briefly review the mechanism underlying the formation 

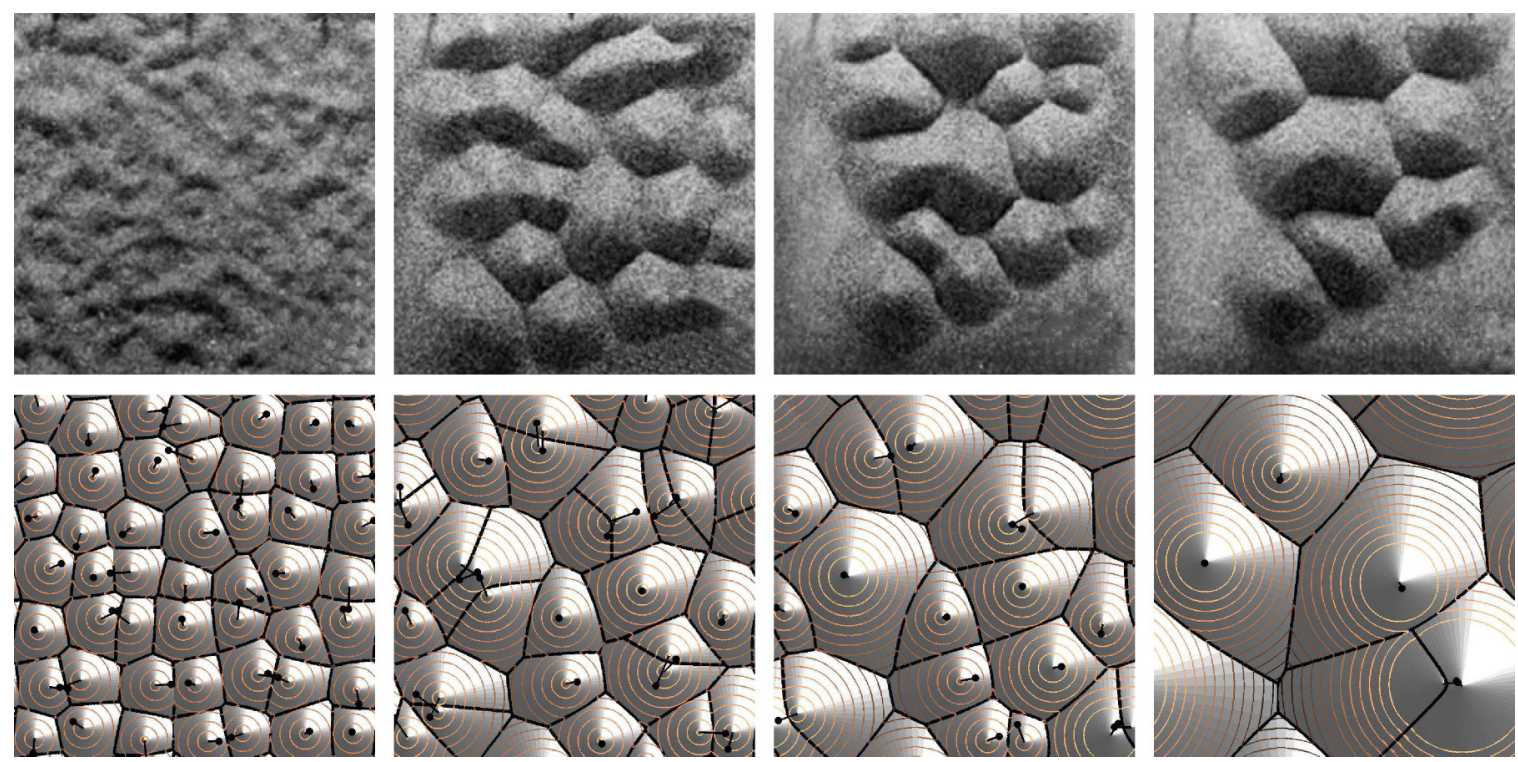

FIG. 1. (Color online) Top view of coarsening Faraday heaps in experiment (top row, images courtesy of Shinbrot [12]) and in the model of the present paper (bottom row). Heaps move towards each other until they merge. In the experimental snapshots this has been marked by gray lines; in the model we indicate the velocity of the heaps by black lines (originating from the peaks of the heaps). The equidistant height lines illustrate the fact that the slope angle $\alpha$ is everywhere the same; in the present simulations $\alpha=18.5$ degrees, in agreement with experiments on millimeter-sized glass beads [11]. An animation is provided as Supplemental Material [29].

of heaps. For a detailed discussion we refer to van Gerner et al. [11] and references therein. Central is the air flow induced by the partial vacuum that is created under the granular layer when it detaches from the plate. Suppose a small heap, with only a slight slope, is present due to some random fluctuation. Since the air flow enters the bed perpendicularly to the slopes of the heap, its velocity has a slight horizontal component, dragging the particles towards the center of the heap. From here they go upwards (where the bed is less dense), hence increasing the slope, so that in the next cycle the inward motion will be somewhat stronger. The slope of the heap will continue to grow until the inward motion is balanced by the avalanching of particles down the sides of the heaps. This equilibrium state - which is attained within a few seconds-sets the final angle of the heap. Hence, very quickly after the plate is set into vibration, one observes a landscape of many small heaps, all with the same slope $\alpha$ (typically around 20 degrees).

The horizontal positions of the peaks are not fixed though: Since one side will always be larger than the other, the air drag from that side is dominant and the peak will thus move in the direction of the smallest side. In this process, heaps will meet and then merge, since when they start to overlap, the area of the sides that are touching quickly diminishes, which causes the peaks to be pushed towards each other. The angle of the slopes $(\alpha)$ remains the same during the whole coarsening process. It is this insight that lies at the heart of our model.

\section{B. A vertical cut through the landscape}

For clarity, we first consider a vertical cut through a heap [see Fig. 2(a)]. The horizontal drag force due to the air coming in from the left is proportional to the projected slope length $l$; the opposed drag force from the right is proportional to the length $r$. These forces act only during a short part $\delta t_{1}$ of the driving period and kick the particles inside the dark gray triangles into motion, which thereby acquire a horizontal momentum $\Delta p_{x}$ :

$$
F_{x} \delta t_{1}=\Delta p_{x}=m u_{x} .
$$

(a)
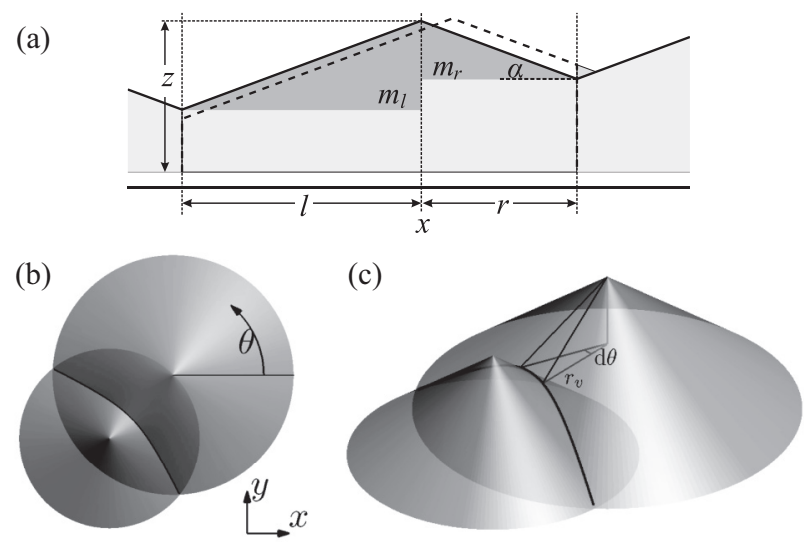

FIG. 2. (a) A vertical cut through a Faraday heap. The dashed profile indicates the position of the heap after one time step $d t$. The dark shading indicates the parts of the heap that are actively engaged in this step of the coarsening process, while the lighter parts are currently inactive and act as a sand reservoir. (b) and (c) Two cones representing two heaps during the coarsening process. The intersection line between the cones is calculated in the appendix. In our model we first determine the intersection lines of each heap with all surrounding heaps, after which the new position and height of the heaps are calculated from Eqs. (4) and (5) under the constraint that the volume of each individual heap remains constant. When two heaps merge, the mass of the resulting heap is simply the sum of the masses of the individual heaps. 
Here $F_{x} \propto(l-r)$ is the net drag force in the horizontal direction and $m \propto l^{2}+r^{2}$ the mass contained in the dark triangles. This mass thus gets a horizontal velocity $u_{x}=$ $u_{x}(l, r) \propto \delta t_{1}(l-r) /\left(l^{2}+r^{2}\right)$.

This velocity is maintained for a time $\delta t_{2}$, during which the particles are in free flight, until the heap collides again with the vibrating plate [3]. Hence, during each complete vibration cycle the top of the heap will be displaced over a distance $\Delta x=u_{x}(l, r) \delta t_{2}$. Since $\delta t_{1}$ and $\delta t_{2}$ are constant, the time rate of change of the horizontal position of a heap (on a time scale much larger than the vibration period, such that the dragging can be viewed as a continuous process) is thus given by

$$
\frac{d x}{d t}=C_{1} \frac{l-r}{l^{2}+r^{2}},
$$

with $C_{1}$ a constant (which can be determined from experimental data [11]) depending on the driving strength and the characteristics of the experimental setup.

\section{The full three-dimensional model}

We now extend the above analysis to the full problem. The change of momentum in the $x$ direction of a small slice $d \theta$ of the heap [see Figs. 2(b) and 2(c)] is proportional to the surface area of the slice multiplied by the component of the unit vector in the $x$ direction $(-\cos \theta)$ :

$$
-\cos \theta \frac{r_{v}^{2}(\theta) d \theta}{2 \cos \alpha} .
$$

Here the surface area of the slice is $\frac{1}{2} r_{v}^{2}(\theta) d \theta / \cos \alpha$, with $\alpha$ the constant slope angle and $r_{v}(\theta)$ the distance between the intersection line and the vertical axis at the peak of the cone. The total change in momentum of a heap in the $x$ direction is found by calculating the intersection lines with all neighboring heaps (see appendix) and integrating the slices $d \theta$ over a complete revolution from $\theta=0$ to $2 \pi$. In order to obtain the change per unit time of the heap position, the total momentum has to be divided by the affected volume $\frac{1}{4} \tan \alpha \int_{0}^{2 \pi} r_{v}^{3}(\theta) d \theta$, which yields for the $x$ direction:

$$
\frac{d x}{d t}=-C_{2} \frac{\int_{0}^{2 \pi} r_{v}^{2}(\theta) \cos \theta d \theta}{\int_{0}^{2 \pi} r_{v}^{3}(\theta) d \theta},
$$

(where the dependence on the slope angle $\alpha$ has been incorporated in the prefactor $C_{2}$ ) and analogously for the $y$ direction:

$$
\frac{d y}{d t}=-C_{2} \frac{\int_{0}^{2 \pi} r_{v}^{2}(\theta) \sin \theta d \theta}{\int_{0}^{2 \pi} r_{v}^{3}(\theta) d \theta}
$$

The associated change of the peak height $d h / d t$ is calculated keeping the volume of the substructure of each heap $\int_{0}^{2 \pi}\left[h-r_{v}(\theta) \tan \alpha\right] \frac{1}{2} r_{v}^{2}(\theta) d \theta$ [cf. the light gray parts in Fig. 2(a)] constant in time. The latter condition is only relaxed in the case of a merging event (i.e., when the distance between two peaks falls below a certain small threshold, the precise value of which has no significant influence on the results): At this moment the volume of the two merging heaps is summed. Finally, knowing the new position and height of each peak we determine the new locations of the intersection lines [30].
In a typical evaluation of the coarsening model (see Fig. 1, bottom row) we start with an initial condition where the distances between the peaks in the $x$ and $y$ direction are taken from a uniform distribution between $0.5 \mathrm{~L} / \sqrt{N_{0}}$ and $1.5 L / \sqrt{N_{0}}$, with $L$ the dimension of the (square) domain and $N_{0}$ the initial number of heaps. The heights of the peaks are distributed uniformly over the interval $z_{0} \pm 0.05 \mathrm{~L} / \sqrt{N_{0}}$. The domain has cyclic boundary conditions. The four snapshots in the bottom row of Fig. 1 show the evolution of a heap pattern (with initially $N_{0}=7 \times 7=49$ heaps) obtained by numerically evaluating the model. The coarsening behavior resembles that of the experimental photos in the top row.

There is just one visible difference between the two evolutions. In the experiment we discern the gradual formation of a depleted outer zone (where hardly any sand grains are left) around the heaps, whereas such a zone is absent in the simulation. This has to do with the total amount of sand in the system, which in the experiment is less than in the depicted simulation. We will come back to the role of depletion in Sec. IV.

\section{SCALING LAW: COARSENING WITHOUT DEPLETION}

\section{A. Average time for neighboring heaps to merge}

To study the statistics of the coarsening behavior we carried out 1000 runs of the model, each time starting with $N_{0}=$ $225(=15 \times 15)$ heaps. The black dots in Fig. 3 show the averaged lifetime of the $N$-heap state $\tau_{N}$ as a function of $N$. It is seen that the model yields an algebraic scaling with $\tau_{N}$ being proportional to $N^{-2}$.

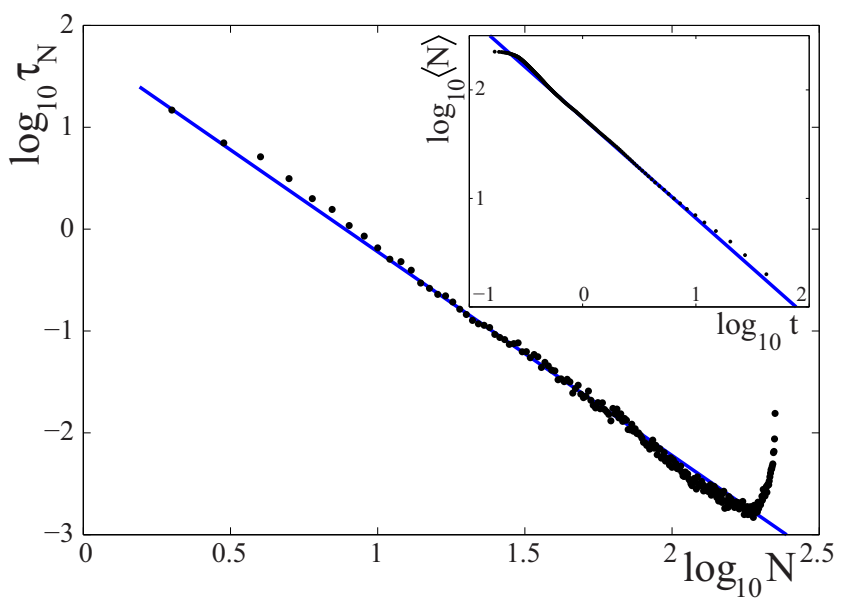

FIG. 3. (Color online) Coarsening without depletion. Mean life time of the $N$-heap state $\tau_{N}$ as a function of the number of heaps $N$ in a doubly logarithmic plot. The black dots are our numerical data averaged over 1000 runs of the model, each time starting with $15 \times 15=225$ heaps. The blue line is the theoretically predicted scaling behavior $\tau_{N} \propto N^{-2}$. The deviations from this power law at large values of $N$ are a start-up effect due to our specific choice of the initial state, as explained in the text. The inset shows how the number of heaps, averaged over all runs, decreases with time. The blue line has a slope of -0.93 , close to the predicted value of -1 corresponding to $\langle N(t)\rangle \propto t^{-1}$. 
This scaling can be understood if we nondimensionalize $x, y$, and $r_{v}(\theta)$ in Eqs. (4) and (5), measuring them relative to the average heap size $L / \sqrt{N}$ (i.e., $\tilde{x}=x \sqrt{N} / L$ and $\widetilde{r}_{v}=$ $\left.r_{v} \sqrt{N} / L\right)$. This yields

$$
\frac{d \widetilde{x}}{d t}=-\frac{N}{L^{2}} C_{2} \frac{\int_{0}^{2 \pi} \widetilde{r}_{v}^{2}(\theta) \cos \theta d \theta}{\int_{0}^{2 \pi} \widetilde{r}_{v}^{3}(\theta) d \theta},
$$

and analogously for $d \tilde{y} / d t$. That is, the change in the dimensionless position of the peak (given by $d \tilde{x} / d t$ and $d \tilde{y} / d t$ ) scales linearly with $N$. The average time for two heaps to merge in the $N$-heap state, $T_{\text {av }}(N)$, will therefore scale as $1 / N$.

This argument can be further strengthened by nondimensionalizing also the time variable $\tilde{t}=\left(N C_{2} / L^{2}\right) t$, after which Eq. (6) takes the following fully dimensionless form:

$$
\frac{d \widetilde{x}}{d \widetilde{t}}=-\frac{\int_{0}^{2 \pi} \widetilde{r}_{v}^{2}(\theta) \cos \theta d \theta}{\int_{0}^{2 \pi} \widetilde{r}_{v}^{3}(\theta) d \theta},
$$

and analogously for $d \tilde{y} / d \widetilde{t}$. Equation (7) contains no reference anymore to the number of heaps in the system: The normalization has turned any $N$-heap state into the same standard state in which the average diameter of the sand heaps is equal to 1 , and if one calculates the average time it takes for two sand heaps to merge (in this standard state) one obtains an answer, say $\widetilde{T}_{\text {av }}$, that is necessarily independent of $N$. This means, with $t=\left(L^{2} / N C_{2}\right) \tilde{t}$, that the duration in ordinary dimensional time is $T_{\mathrm{av}}(N) \propto \widetilde{T}_{\mathrm{av}} / N \propto 1 / N$, in full agreement with what we had already concluded on the basis of Eq. (6).

\section{B. Lifetime of the $N$-heap state}

The life expectancy or "lifetime" of the $N$-heap state $\left(\tau_{N}\right)$ is not equal to the average merging time $T_{\mathrm{av}}(N)$, but to the shortest merging time from all pairs of heaps in the system, since it is the first merging event that terminates the $N$-heap state.

When $N$ is not too small, the merging events may be assumed to be statistically independent and the corresponding distribution of merging times $T_{i}(i=1, \ldots, N)$ will be exponential: $P\left(T_{i}\right)=P(0) e^{-T_{i} / T_{\text {av }}}$. We checked that this is indeed the case in our system [31]. A property of the exponential distribution is that the value of the smallest element $\min \left\{T_{1}, . ., T_{N}\right\}$ (alias $\tau_{N}$ ) is inversely proportional to the sample length $N$ :

$$
\tau_{N}=\min \left\{T_{1}, . ., T_{N}\right\}=\frac{T_{\mathrm{av}}(N)}{N},
$$

and, together with our earlier result $T_{\mathrm{av}}(N) \propto 1 / N$ this leads to the following algebraic scaling law:

$$
\tau_{N} \propto \frac{1}{N^{2}} .
$$

This is exactly the behavior we observe in our model (see Fig. 3).

The fact that initially (i.e., for large $N$ ) the numerical data do not yet follow the power law (9) is because we start out from a state in which all 225 heaps are of roughly equal size, which is quite different from the distribution the system will quickly evolve to (and which has been assumed to hold in the above derivation, when we took the merging events to be statistically independent). Using a different initial heap distribution (e.g., a normal distribution) gives a different picture during the early stages, but again it soon converges to the distribution that complies with the power law scaling [31].

Heaps of similar size (and thus similar slope lengths all around) are much less mobile than dissimilar ones, hence they are much slower to merge; therefore the measured lifetimes during the initial stages are considerably larger than what is predicted by the power law.

Evidently, the power law (9) must also lose its validity at the very end of the coarsening process: For $N=1\left(\log _{10} N=0\right)$ the lifetime $\tau_{N}$ becomes infinite because the 1-heap state is stable. That is why the straight blue line in Fig. 3 has been cut short before it reaches the vertical axis at $\log _{10} N=0$.

\section{How the number of heaps decreases with time}

From the scaling law (9) we can directly deduce how the number of heaps $N(t)$ decreases with time towards the end of the coarsening process. It suffices to observe that the total elapsed time at the instant when the $N$-heap state ends, $t(N)$, is equal to the sum of all $\tau_{N^{\prime}}$ from $N^{\prime}=N_{\text {in }}$ (the initial number of heaps) to $N^{\prime}=N$ :

$$
\begin{aligned}
t(N) & =\sum_{N_{\text {in }}}^{N} \tau_{N^{\prime}} \approx \int_{N}^{N_{\text {in }}} \tau_{N^{\prime}} d N^{\prime} \\
& \propto \int_{N}^{N_{\text {in }}} \frac{d N^{\prime}}{N^{\prime 2}}=K\left(\frac{1}{N}-\frac{1}{N_{\text {in }}}\right),
\end{aligned}
$$

where $K$ is a constant prefactor. For $N \ll N_{\text {in }}$ (i.e., towards the end of the coarsening process) the above relation simplifies to $t(N) \approx K / N$, or equivalently,

$$
\langle N(t)\rangle \propto 1 / t \quad\left(\text { when }\langle N(t)\rangle \ll N_{\text {in }}\right) .
$$

Here we write $\langle N(t)\rangle$ (where the angles denote the ensemble average) rather than $N(t)$ itself, because it is understood that for a single realization of the experiment there may be quite some statistical spread around this behavior. Thus Eq. (11) should be interpreted as a statistical prediction for the ensemble-averaged number of heaps $\langle N(t)\rangle$, and it is this quantity which is plotted in the inset of Fig. 3. We indeed observe a clear power-law behavior with an exponent of -0.93 , close to the value -1 predicted by Eq. (11). It has to be noted that Eq. (11) is approximate, and only becomes accurate in the limit of very large $N_{\text {in }} \gg N \gg 1$. The discrepancy between the measured slope $(-0.93)$ and the predicted one $(-1)$ can be traced back to these approximations.

\section{Average heap size as a function of time}

One of the quantities of experimental interest, apart from $\tau_{N}$ and $N(t)$, is the size of the heaps, represented by the ensembleaveraged radius $\langle r(t)\rangle$. This quantity is readily derived from the above scaling law. Assuming that the initial layer of sand is sufficiently thick - such that there will never be regions that are completely devoid of sand-the total projected area of all heaps (rather than their volume) is a conserved quantity, say $A_{\text {tot }}$. Naturally, the volume of the whole bed is conserved; however, the part of this volume that actively participates in the coarsening process (i.e., the volume corresponding to the dark 
triangles in Fig. 2) changes with time, drawing sand (whenever required) from the lower layers of the bed that act as a reservoir.

The ensemble-averaged area per heap in the $N$-heap state is simply

$$
\langle A(t)\rangle=\frac{A_{\text {tot }}}{\langle N(t)\rangle},
$$

and the ensemble-averaged radius $\langle r(t)\rangle$ scales as the square root of this area, so

$$
\langle r(t)\rangle \propto\left(\frac{A_{\mathrm{tot}}}{\langle N(t)\rangle}\right)^{1 / 2} \propto\langle N(t)\rangle^{-1 / 2} \propto t^{1 / 2},
$$

where in the last step we have used Eq. (11). Since the latter proportionality $[\langle N(t)\rangle \propto 1 / t]$ becomes increasingly accurate towards the end of the coarsening process, when $\langle N(t)\rangle \ll N_{\text {in }}$, the same holds for the above scaling law $\langle r(t)\rangle \propto t^{1 / 2}$.

Indeed, when we plot the ensemble-averaged square root of the heap area $\langle\sqrt{A(t)}\rangle$, which is proportional to $\langle r(t)\rangle$, we retrieve the $t^{1 / 2}$ power law over two decades: The black dots in the doubly logarithmic plot of Fig. 4(b) follow a straight line with a slope close to $1 / 2$. The best fit to these data (indicated by the solid red line) provides a slope of 0.46 . Not incidentally, this is half of the absolute value of -0.93 that was found for $\langle N(t)\rangle$ (in the inset of Fig. 3).

The scaling law ceases to hold in the 1-heap state, when the final heap reaches the maximum size (dictated by the size of the system) and from this point on its radius simply remains constant; see the black data set in Fig. 4(b) for large $t$. In the presence of depletion [represented by the other data sets in Fig. 4(b)] the maximum size is smaller because in this case there is a limited amount of sand in the system, and also because the typical (ensemble-averaged) end state may consist of several isolated heaps. We come back to this in the next section.

\section{THE ROLE OF DEPLETION}

\section{A. Experimental and numerical observations}

If the initial layer of sand is relatively thin, in the course of the coarsening process one will witness the appearance of depleted zones, where the bare vibrating plate becomes visible. We already encountered this phenomenon in the experimental snapshots of Fig. 1 (top row). The depletion has a profound effect on the coarsening dynamics. Shinbrot [12] found experimentally that in this case $\langle r(t)\rangle \propto t^{0.32 \pm 0.03}$ [12], i.e., a considerably slower growth than the $t^{1 / 2}$ behavior of the nondepleted system. This stands to reason, since the heaps in the latter case never feel any shortage of sand (they float, so to speak, on a reservoir of sand that supplies them with all the extra grains they need) whereas in the presence of depletion the growth is restricted by the limited supply of sand.

We will study the influence of the depletion by starting from a finite, noncyclic configuration of connected heaps. The initial condition is prepared in the same way as for the cyclic system described in Sec. II. When depleted zones start to appear, the projected area of the collection of heaps is evidently not conserved anymore. The conserved quantity now becomes the total volume $V_{\text {tot }}$ of the heaps (i.e., all the sand actively participating in the coarsening process), since the "reservoir"

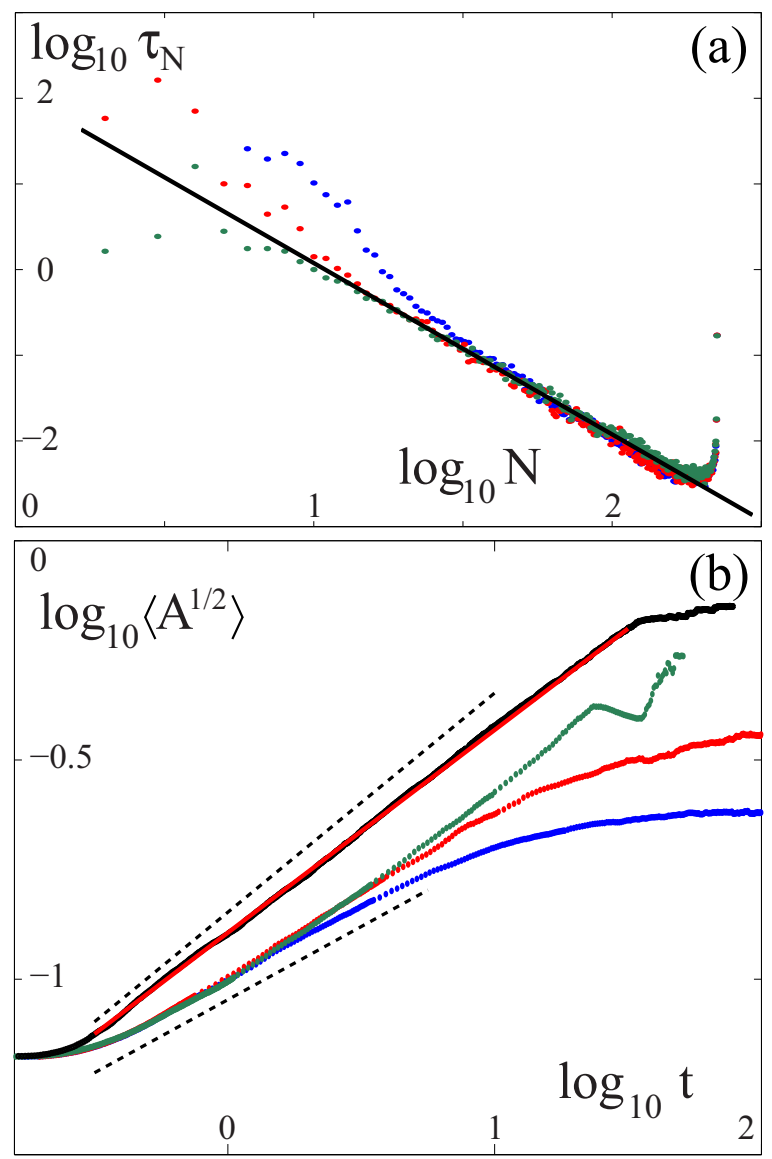

FIG. 4. (Color online) Coarsening for various degrees of depletion. (a) Average lifetime $\tau_{N}$ of the $N$-heap state for three systems with depletion parameters $\Phi_{\text {sup }}=0.57$ (green), 0.35 (red), and 0.28 (blue) as a function of $N$ in a doubly logarithmic plot. The black line indicates the $N^{-2}$ power law of the nondepleted system with $\Phi_{\text {sup }}=1$. (b) Growth of the average heap size $\langle\sqrt{A(t)}\rangle$ for the same three systems (in comparison with the data for the nondepleted system, black symbols), again in a doubly logarithmic plot. The dashed lines indicate power laws with exponents $1 / 3$ (lower line) and 1/2 (upper line), respectively. The linear fit to the data for the nondepleted system (the solid red line through the black data) has a slope of 0.462 .

of sand below the dark triangles of Fig. 2 has been emptied and all the sand is contained in the actual heaps. Depletion can be incorporated in the numerical model without any problem, simply by starting out with a thinner layer of sand. The only (minor) change is that apart from intersection lines between two cones (see Appendix); in this case one also has to deal with intersection lines between a cone and the flat floor.

In this situation, the coarsening process is no longer guaranteed to run its full course until all the sand is contained in one single heap; it can also come to a halt in a state with multiple heaps, if these happen to get isolated from each other without any overlap. For convenience, however, we will link the total volume to the 1-heap state (whether it is reached or not) in which the entire volume $V_{\text {tot }}$ is contained in a single heap. This is a perfectly symmetric conical heap (recall that the slope angle $\alpha$ is the same in all directions), the radius of which we call $r_{1}$; it is related to the volume via $V_{\text {tot }}=\frac{1}{3} \pi r_{1}^{3} \tan \alpha$.

Each system can be characterized by the ratio $\Phi_{\text {sup }}$ of the ground surface of the single cone containing all the sand 
$\left(\pi r_{1}^{2}\right)$ and the area covered by the $N_{\text {in }}$ heaps in the initial state:

$$
\Phi_{\text {sup }}=\frac{\pi r_{1}^{2}}{\text { initial area covered }} .
$$

This fraction is equal to 1 in the case without depletion, whereas $\Phi_{\text {sup }}<1$ in all cases where a certain degree of depletion occurs.

In Fig. 4 we plot the average lifetime $\tau_{N}$ of the $N$-heap state versus $N$ and the time evolution of the average heap size $\left\langle A^{1 / 2}\right\rangle$ for three different values of $\Phi_{\text {sup }}$, namely $0.57,0.35$, and 0.28 . In each case we performed about 100 runs starting from $N_{0}=225$ initial heaps. Figure 4(a) shows that for large $N$ the systems still follow the $\tau_{N} \propto N^{-2}$ power law, but begin to deviate towards the end, when the depletion effects come into play. As expected, the smaller the value of $\Phi_{\text {sup }}$ (i.e., stronger depletion) the sooner the discrepancy makes it appearance. For $\Phi_{\text {sup }}=0.28$ the scaling $\tau_{N} \propto N^{-2}$ is already lost at the level of 20 heaps, whereas for $\Phi_{\text {sup }}=0.57$ it remains valid until the 10-heap state is reached.

For $\Phi_{\text {sup }}=0.57$, despite the depleted zone, the heaps never entirely lose contact with each other and the end result is still 1 single heap. In the cases $\Phi_{\text {sup }}=0.35$ and 0.28 , however, the process practically always ends up with a set of stable isolated heaps. For $\Phi_{\text {sup }}=0.35$ the final state consists of 3.8 heaps on average, and for $\Phi_{\text {sup }}=0.28$ this number has gone up to 8.5. The associated curves in Fig. 4(b) converge to constant values for large $t$, corresponding to the average radius of the heaps in the final state.

In the same Fig. 4(b) we see that the heap sizes for $\Phi_{\text {sup }}<1$ no longer follow the $t^{1 / 2}$ of the nondepleted system (represented by the black dots of the upper data set) but instead show an intermediate regime in which they grow as $t^{1 / 3}$. This is in quantitative agreement with Shinbrot's experimental finding mentioned above, according to which the size grows as $\langle r(t)\rangle \propto t^{0.32 \pm 0.03}$.

\section{B. Explanation of the observed scaling behavior}

In order to explain this $t^{1 / 3}$ power law we consider an $N$-heap state (with ensemble-averaged heap radius $\left\langle r_{N}\right\rangle$ ) in a stage of the coarsening process when there is no inactive "reservoir" anymore, so all the sand in the system is actively participating. Under these circumstances, the total volume of the $N$ heaps equals the volume of the single conical heap of radius $r_{1}$. This volume conservation means that $N\left\langle r_{N}^{3}\right\rangle=r_{1}^{3}$, or equivalently (with $\left\langle r_{N}^{3}\right\rangle=\left\langle r_{N}\right\rangle^{3}$ ),

$$
\left\langle r_{N}\right\rangle=r_{1} N^{-1 / 3} \text {. }
$$

Thus $\langle r(t)\rangle \propto\langle N(t)\rangle^{-1 / 3}$ and if we apply Eq. (11) this immediately leads to $\langle r(t)\rangle \propto t^{1 / 3}$, which is the same result as obtained from Fig. 4(b).

This last step, applying the relation $\langle N(t)\rangle \propto t^{-1}$ of Eq. (11), deserves some special care, since this relation hinges on the validity of the scaling of the lifetimes as $\tau_{N} \propto N^{-2}$. As we have seen in Fig. 4(a), this validity breaks down towards the end of the coarsening process. So on the one hand, we require that the $N$-heap state under consideration is in a sufficiently advanced stage of the coarsening process for the "reservoir" to be emptied [and to ensure that $\langle N(t)\rangle \ll N_{\text {in }}$, which allows us to use Eq. (11)] and on the other hand we must avoid the final stages of the process (because here the scaling $\tau_{N} \propto N^{-2}$ loses its validity and hence also Eq. (11) cannot be expected to hold anymore). The combination of both conditions defines the intermediate interval during which the $t^{1 / 3}$ scaling is valid. In Fig. 4(b) we see that this interval covers about one decade around $\log t=2$.

\section{CONCLUSION}

We have shown that the coarsening of Faraday heaps, despite the fact that it involves a complex interplay between air drag forces on the particles in the interior of the heaps and avalanching along the slopes, can successfully be captured in a relatively simple model. At the basis of this model lie two crucial observations: (i) The slope $\alpha$ of the heaps is constant throughout the entire process and (ii) the drag forces that set the heaps into motion scale with the area of the heap flanks.

From our model it can be deduced that the mean life time of the $N$-heap state, as long as the heaps make contact with neighboring heaps on each side, scales as $\tau_{N} \propto N^{-2}$. This result implies, with increasing accuracy towards the end of the coarsening process, that the (ensemble-averaged) number of heaps decays inversely proportional to the total time elapsed since the start of the experiment: $\langle N(t)\rangle \propto 1 / t$.

For systems with an ample amount of sand, which never develop depleted regions, we have demonstrated that the ensemble-averaged radius $\langle r(t)\rangle$ of the heaps grows as $t^{1 / 2}$. In this case the coarsening always ends up with a single heap on a sublayer of sand.

If the amount of sand is less, such that toward the end of the coarsening process regions appear which are devoid of sand, $\langle r(t)\rangle$ grows more slowly, namely as $t^{1 / 3}$, in agreement with experimental observations [12]. For severely depleted systems, in which the heaps at some point lose contact with each other, the growth stops altogether and the coarsening is arrested in a state with multiple isolated, conical heaps.

Intriguingly, the above laws coincide precisely with two main universality classes of how length scales grow in a multitude of coarsening, aging, and ripening processes in nature. For processes in which the order parameter is a nonconserved quantity one generically finds that the length scale grows as $L(t) \propto t^{1 / 2}$, whereas for the more special case in which the order parameter is conserved one may anticipate on general grounds that $L(t) \propto t^{1 / 3}$ [32]. This suggests that the volume of sand contained in the active parts of the heaps (which is nonconserved in the case without depletion, and conserved in the presence of depletion) may be identified as the proper order parameter for Faraday heaping. It will be interesting and highly relevant to further investigate this link with the modern theory of phase transitions and aging processes in nonequilibrium systems [33-36].

\section{ACKNOWLEDGMENTS}

We wish to thank Detlef Lohse for inspiring and insightful discussions. This work is part of the research program of the Stichting FOM, which is financially supported by the Netherlands Organisation for Scientific Research (NWO). K.vdW. cordially thanks his colleagues at the Physics of Fluids group of the University of Twente, The Netherlands, for their 
kind hospitality during several extended work visits. K.vdW. also acknowledges partial financial support from the research project "MACOMSYS," Grant No. MIS 379337, co-financed by the European Union (European Social Fund) and Greek national funds through the Operational Program "Education and Lifelong Learning" of the National Strategic Reference Framework-Research Funding Program THALES: Investing in knowledge society through the European Social Fund.

\section{APPENDIX: HOW THE INTERSECTION LINE BETWEEN TWO CONES IS CALCULATED}

In this Appendix we outline the method by which we calculate the intersection line between two cones, since this is an important step in the analysis. Just as in the main text, we freely switch between Cartesian and polar coordinates $(x=r \cos \theta, y=r \sin \theta)$ as the occasion demands.

Consider two conical heaps with their peaks (of height $h_{1}$ and $\left.h_{2}\right)$ located above the ground points $\left(x_{1}, y_{1}\right)$ and $\left(x_{2}, y_{2}\right)$, respectively, and slope angle $\alpha$ all around. In Cartesian coordinates, these cones are given by the equation:

$$
\left(x-x_{i}\right)^{2}+\left(y-y_{i}\right)^{2}=\frac{\left(z-h_{i}\right)^{2}}{\tan ^{2} \alpha}, \quad i=1,2 .
$$

In polar coordinates, taking $\left(x_{1}, y_{1}\right)=(0,0)$, the equation for the first cone is simply

$$
r^{2}=\frac{\left(z-h_{1}\right)^{2}}{\tan ^{2} \alpha}
$$

Now let $r_{12}$ denote the distance between the origins of the first and second cone and $\theta_{12}$ the corresponding angle, then $\left(x_{2}, y_{2}\right)=\left(r_{12} \cos \theta_{12}, r_{12} \sin \theta_{12}\right)$ and the equation for the second cone in polar coordinates becomes

$\left(r \cos \theta-r_{12} \cos \theta_{12}\right)^{2}+\left(r \sin \theta-r_{12} \sin \theta_{12}\right)^{2}=\frac{\left(z-h_{2}\right)^{2}}{\tan ^{2} \alpha}$,

or equivalently,

$$
r^{2}+r_{12}^{2}-2 r r_{12} \cos \left(\theta-\theta_{12}\right)=\frac{\left(z-h_{2}\right)^{2}}{\tan ^{2} \alpha} \text {. }
$$

Solving Eq. (A2) for $z$ and substituting this result in Eq. (A4) yields

$r^{2}+r_{12}^{2}-2 r r_{12} \cos \left(\theta-\theta_{12}\right)-\left(r+\frac{h_{1}}{\tan \alpha}-\frac{h_{2}}{\tan \alpha}\right)^{2}=0$,

which is a quadratic equation for $r$ and hence directly yields the radial coordinate $r$ of the intersection line as a function of the angle $\theta$ [which in the main text is called $r_{v}(\theta)$ ]:

$$
r=\frac{r_{12}^{2} \tan ^{2} \alpha-\left(h_{2}-h_{1}\right)^{2}}{2 \tan \alpha\left[r_{12} \tan \alpha \cos \left(\theta-\theta_{12}\right)+h_{2}-h_{1}\right]} .
$$

Repeating this procedure for all the surrounding heaps provides us with the set of intersection lines necessary for the calculation of Eqs. (4) and (5).
[1] M. Faraday, On a peculiar class of acoustical figures; and on certain forms assumed by a group of particles upon vibrating elastic surfaces, Philos. Trans. R. Soc. London 52, 299 (1831).

[2] B. Thomas and A. M. Squires, Support for Faraday's View of Circulation in a Fine-Powder Chladni Heap, Phys. Rev. Lett. 81, 574 (1998).

[3] H. J. van Gerner, M. A. van der Hoef, D. van der Meer, and K. van der Weele, Interplay of air and sand: Faraday heaping unravelled, Phys. Rev. E 76, 051305 (2007).

[4] P. Evesque and J. Rajchenbach, Instability in a Sand Heap, Phys. Rev. Lett. 62, 44 (1989).

[5] C. Laroche, S. Douady, and S. Fauve, Convective flow of granular masses under vertical vibrations, J. Phys. 50, 699 (1989).

[6] H. K. Pak, E. van Doorn, and R. P. Behringer, Effects of Ambient Gases on Granular Materials under Vertical Vibration, Phys. Rev. Lett. 74, 4643 (1995).

[7] T. Akiyama, K. M. Aoki, K. Yamamoto, and T. Yoshikawa, Experimental study on vibration-induced convection and heaping in granular beds, Gran. Matter 1, 15 (1998).

[8] K. Kumar, E. Falcon, K. M. S. Bajaj, and S. Fauve, Shape of convective cell in Faraday experiment with fine granular materials, Physica A 270, 97 (1999).

[9] R. J. Milburn, M. A. Naylor, A. J. Smith, M. P. Leaper, K. Good, M. R. Swift, and P. J. King, Faraday tilting of water-immersed granular beds, Phys. Rev. E 71, 011308 (2005).

[10] E. van Doorn and R. P. Behringer, Onset and evolution of a wavy instability in shaken sand, Phys. Lett. A 235, 469 (1997).
[11] H. J. van Gerner, G. Caballero, D. van der Meer, K. van der Weele, and M. A. van der Hoef, Coarsening of Faraday Heaps: Experiment, Simulation, and Theory, Phys. Rev. Lett. 103, 028001 (2009).

[12] T. Shinbrot, Granular coarsening, Gran. Matter 1, 145 (1998).

[13] A. Stegner and J. E. Wesfreid, Dynamical evolution of sand ripples under water, Phys. Rev. E 60, R3487 (1999).

[14] J. L. Hansen, M. van Hecke, A. Haaning, C. Ellegaard, K. H. Andersen, T. Bohr, and T. Sams, Pattern formation: Instabilities in sand ripples, Nature (London) 410, 324 (2001).

[15] K. H. Andersen, M.-L. Chabanol, and M. van Hecke, Dynamical models for sand ripples beneath surface waves, Phys. Rev. E 63, 066308 (2001).

[16] K. H. Andersen, M. Abel, J. Krug, C. Ellegaard, L. R. Søndergaard, and J. Udesen, Pattern Formation of Vortex Ripples in Sand: Nonlinear Modeling and Experimental Validation, Phys. Rev. Lett. 88, 234302 (2002).

[17] E. K. O. Hellén and J. Krug, Coarsening of sand ripples in mass transfer models with extinction, Phys. Rev. E 66, 011304 (2002).

[18] T. Schnipper, K. Mertens, C. Ellegaard and T. Bohr, Amplitude equation and long-range interactions in underwater sand ripples in one dimension, Phys. Rev. E 78, 047301 (2008).

[19] R. A. Bagnold, The Physics of Blown Sand and Desert Dunes (Methuen, London, 1954).

[20] P. Hersen, S. Douady, and B. Andreotti, Relevant Length Scale of Barchan Dunes, Phys. Rev. Lett. 89, 264301 (2002).

[21] E. J. R. Parteli and H. J. Herrmann, A simple model for a transverse dune field, Physica A 327, 554 (2003). 
[22] V. Schwämmle and H. J. Herrmann, Solitary wave behavior of sand dunes, Nature (London) 426, 619 (2003).

[23] P. Hersen, K. H. Andersen, H. Elbelrhiti, B. Andreotti, P. Claudin, and S. Douady, Corridors of barchan dunes: Stability and size selection, Phys. Rev. E 69, 011304 (2004).

[24] P. Claudin and B. Andreotti, A scaling law for Aeolian dunes on Mars, Venus, Earth, and for subaqueous ripples, Earth Planet. Sci. Lett. 252, 30 (2006).

[25] B. Andreotti, A. Fourrière, F. Ould-Kaddour, B. Murray, and P. Claudin, Giant Aeolian dune size determined by the average depth of the atmospheric boundary layer, Nature (London) 457, 1120 (2009).

[26] M. C. Cross and P. C. Hohenberg, Pattern formation out of equilibrium, Rev. Mod. Phys. 65, 851 (1993).

[27] H. M. Jaeger, S. R. Nagel, and R. P. Behringer, Granular solids, liquids, and gases, Rev. Mod. Phys. 68, 1259 (1996).

[28] I. S. Aranson and L. S. Tsimring, Patterns and collective behavior in granular media: theoretical concepts, Rev. Mod. Phys. 78, 641 (2006).

[29] See Supplemental Material at http://link.aps.org/supplemental/ 10.1103/PhysRevE.92.042203 for an animation of the coarsening process as shown in the bottom row of Fig. 1
[30] With an eye to the consistency of the iterative procedure, we note that the shift of mass from one heap into another due to the shift of the intersection lines is of the order of the square of the displacement and therefore vanishes in the limit of zero displacement.

[31] H. J. van Gerner, Newton vs Stokes: Competing forces in granular matter, Ph.D. thesis, University of Twente, 2009 [http://purl.org/utwente/61070].

[32] A. J. Bray, Theory of phase-ordering kinetics, Adv. Phys. 43, 357 (1994).

[33] J. Krug, Origins of scale invariance in growth processes, Adv. Phys. 46, 139 (1997).

[34] M. Henkel, H. Hinrichsen, and S. Lübeck, Non-Equilibrium Phase Transitions, Vol. I: Absorbing Phase Transitions, Series Theoretical and Mathematical Physics (Springer, Dordrecht, 2008).

[35] M. Henkel and M. J. F. Pleimling, Non-Equilibrium Phase Transitions, Vol. II: Ageing and Dynamical Scaling far from Equilibrium, Series Theoretical and Mathematical Physics (Springer, Dordrecht, 2010).

[36] P. L. Krapivsky, S. Redner, and E. Ben-Naim, A Kinetic View of Statistical Physics (Cambridge University Press, Cambridge, 2010). 